\title{
Application of SVM-Based Relevance Feedback in Image Retrieval
}

\author{
Xian.Wei $\mathrm{WU}^{1,}$, Wen.Yang $\mathrm{Yu}^{1}$ and Yu.Bin Yang ${ }^{1}$ \\ ${ }^{1}$ Ningbo Dahongying University, Ningbo 315000, China; \\ awxw786@qq.com
}

Keywords: SVM, relevance feedback, image retrieval.

\begin{abstract}
In the page, we discuss relevance feedback techniques in image retrieval system, and then focus on the SVM-based relevance feedback. Using SVM-based relevance feedback, the precision rate of image retrieval system is satisfied. In order to better understand the effectiveness of SVM-based relevance feedback in image retrieval system, some well-designed experiments are taken out.
\end{abstract}

\section{Introduction}

With the rapid development of multimedia technology and computer network, the application of image information is becoming more and more widely, the large-scale image database managements become an urgent need to solve the problem effectively, the flexible, efficient and accurate image retrieval strategy is one of the key technologies to solve this problem.

Image retrieval[1] is a search process in image feature space. For different users, the need is different also. We know the lower visual characteristics such as color, texture, shape are widely in image retrieval system, but lower visual characteristics are just only lower visual characteristics, not always accord with user's needs, so bring in the user's interaction in image retrieval process is very necessary and important.

At present, in the content-based image retrieval system the extraction of image feature is basically a low-level visual features[2] of images, is far away from the actual semantics of images, it's hard to understand the content of image by using low-level visual features. Therefore, no matter what kind of feature is adopted, no matter what kind of similarity measurement is used, eventually it is the actual user to determine whether two images are similar. Because the focus is different, different user has different standards of image similarity judgment. Therefore need to study using user's interaction how to make the system automatically adapt to the specific requirements, so as to realize better query result.

\section{Relevance feedback techniques}

The Relevance feedback[3] is a powerful way to improve system query result. Its purpose is to learn, discover and capture the user's actual query intention and correctly adjust query strategies in conform with the user's actual demand from user's interaction with the query system. Using relevance feedback, system query strategy can be modified in real time, the adaptive of image retrieval system is strengthen.

Relevance feedback retrieval is a stepwise refinement process, there is a feature adjustment cycle, re-matching, as shown in Fig 1: first, the user is given a query image, the system according to certain algorithm to extract visual characteristics, and then according to the initial weights and certain similarity measurement method to retrieve a series of similar images, the results in ascending order of the distance, this is called "first retrieval", if meet user requirements, retrieve the end; Otherwise, the user to evaluate the results of each image, mark relevant or irrelevant manually, the system according to the user's interaction information to re-calculate the new weight value, and adjust the query vector, and then to re-calculate the similarity, and get the retrieval results, new again into the next state feedback, until meet the user's needs. 


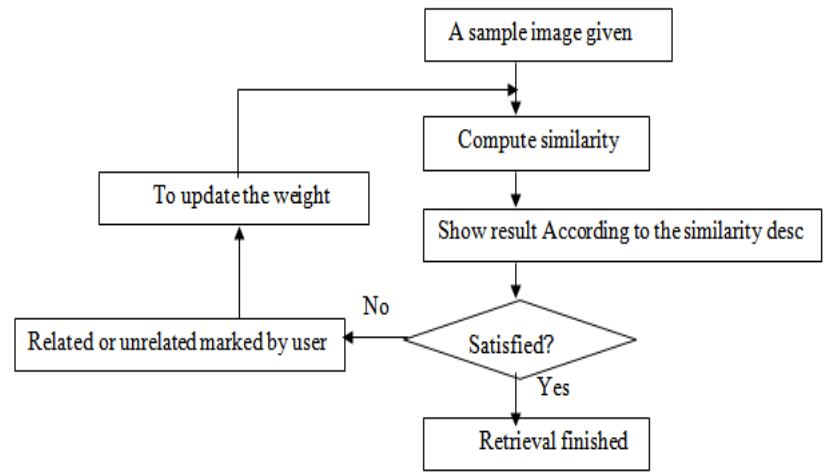

Fig1. Flow of Relevance feedback

\section{The relevance feedback algorithm based on SVM}

With the development of relevant feedback technology, the relevance feedback algorithm based on SVM[4] arises. The basic idea is : sample collection in the retrieval process is a dynamic growing collection, every image in every feedback to the user, can be divided into relevant or irrelevant, then to update the original sample set, through such accumulation, sample collection will gradually meet the requirements of the SVM learning. At the same time, every time after a new feedback, the more satisfied result come into being to achieve the need of user. Through several such feedbacks, user can get desirable result soon.

SVM Algorithm A given linear separable sample $\left(x_{i}, y_{i}\right), i=1,2 \ldots, N, y_{i} \in\{-1,1\}$, assume that a hyper-plane $w \bullet x+b=0$ can separate positive cases and negative cases, we call this hyper-plane classification hyper-plane, the corresponding function is: $g(x)=w \bullet x+b$. There is a best classification plane that not only can separate the two classes but also can maximize the distance of two classes. ${ }^{g(x)}$ after normalization, solving the optimal classification problem is equivalent to minimize

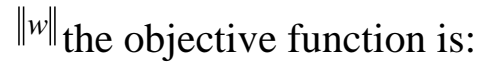

$$
\min \Phi(w)=\frac{\|w\|^{2}}{2}
$$

The constraint conditions of above formula is: $y_{i}\left(w x_{i}+b\right)-1 \geq 0 \quad i=1,2, \ldots, N$.

Define N Larrange operator $\alpha_{i}, i=1,2, \ldots, N$, solving the quadratic optimization problem, you can get the best classification plane, ${ }^{w=\sum_{i=1}^{N} \alpha_{i} y_{i} x_{i}},{ }^{x_{i}}$ which is a sample located in the classification interval planes, are called support vector. The Classification function is:

$$
f(x)=\operatorname{sign}\left(\sum_{i} \alpha_{i} y_{i} x_{i} x+b\right)
$$

Under the condition of the data is not linearly separable, on the one hand, the SVM is introduced into penalty factor $\mathrm{C}$ and the coefficient of relaxation, modifying the objective function is:

$$
\begin{gathered}
\Phi(w)=\frac{1}{2}(w w)+C\left(\sum_{i=1}^{N} \xi_{i}\right) \square \square \square \square \square \square \square \square \square \square \square \square \square \square \square \square \square \square \square \square \square \\
\square \square \square \square \square \square \square \square \square \square \square \square \square \square \square \square \square \square
\end{gathered}
$$

In addition, notice the formula in the formula (2) only appear in the form of dot product, assuming that can map the data to another space $\mathrm{H}$, mapping relationship is as follows: $\psi: R^{d} \rightarrow H$, then the dot product of the formula (2) can transform into the dot product of space $\mathrm{H}$, if found function $\mathrm{K}$ satisfy the Mercer condition, a dot product can be expressed as $K\left(x_{i}, x_{j}\right)=\psi\left(x_{i}\right) \psi\left(x_{j}\right)$, in this function $\mathrm{K}$ is called the kernel function. Then the classification function is changed to :

$$
f(x)=\operatorname{sign}\left(\sum_{i} \alpha_{i} y_{i} K\left(x_{i}, x\right)+b\right)
$$

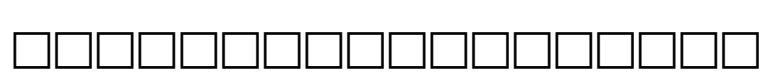

As mentioned above, the nonlinear process of SVM can be summarized as follows: first of all, by nonlinear transformation will transform the input space into a high dimensional space, and then get 
the best linear classification plane in the space, the nonlinear transform is implemented by defining the proper kernel function.

The realization of relevance feedback algorithm based on SVM[5] The basic idea is this: in the retrieval process, allowing users to interact with search results: mark every result image relevant or irrelevant, and then use these user feedback information as a training sample back to the learning system to guide the next round of search, making search results more in line with user needs. After every retrieval, user get $\mathrm{n}$ images according to similarity in descending order, the user mark the images that are relevant with the sample image, these images relevant with the original sample image together constitute a positive example of positive cases of new image collections. Meanwhile the result is returned to those not marked as related to the original image is counter-example image together constitute a new counter-example image collection. Then, these positive and negative examples were SVM learning, you can get a goal on behalf of the user to retrieve the SVM classifier. Using this classification, you can put all images into relevant and irrelevant, then use the associated image set some image retrieval algorithm retrieves and returns the result, and then, mark the result, and so on, until the retrieval results are satisfied.

A detailed learning and retrieval algorithm is as follows:

1) System initialization: first empty the positive set and the negative set.

2) User then selects certain retrieval algorithm for retrieval, and according to the similarity of decreasing order to return to the first $n$ images.

3) User interact with the image retrieval system: mark every result image as relevant or irrelevant, then feedback to the image retrieval system, append the relevant images to the positive set, and append the irrelevant images to the negative set.

4) According to the current state of the positive and negative set, through the SVM learning to make a new SVM classifier, and using the classifier to classify all images, divided into relevant images and irrelevant images.

5) Then among all relevant images, using the original retrieval algorithm for retrieval, and according to the similarity of decreasing order to return to the first $n$ image. If the result is satisfied, end the retrieval process, otherwise go back to step 3.

\section{Experiments and Results}

In order to obtain the performance of relevance feedback technology in image retrieval system, we design two experiments in the paper:

Exp1: In this experiment we use the image database that is selected from "million gallery" (http://www.mypcera.com/photo/photo.htm). The image database has 3300 images and 70 categories, each category contains the number of images ranging from 20 to 60, including 60 categories that the number of images is more than 30. In these categories, we choose eight categories: oceans, peoples, animals, flowers, chairs, mountains, gestures and tools as test image database.

For each retrieval, the system returns 30 images. we take the experiment under the assumption that: if two images are in same category, We think the two images are relevant.

The Experiment is designed as follow: randomly selected 5 images in each category as query image, and then retrieval using color feature, texture feature, shape feature and integrated feature[6] in sequence, and interact with image retrieval system using SVM relevance feedback technique[7]. For every category and every retrieval method, we get 5 precision rates, and record the final average precision rate P, shown in Fig. 2. 


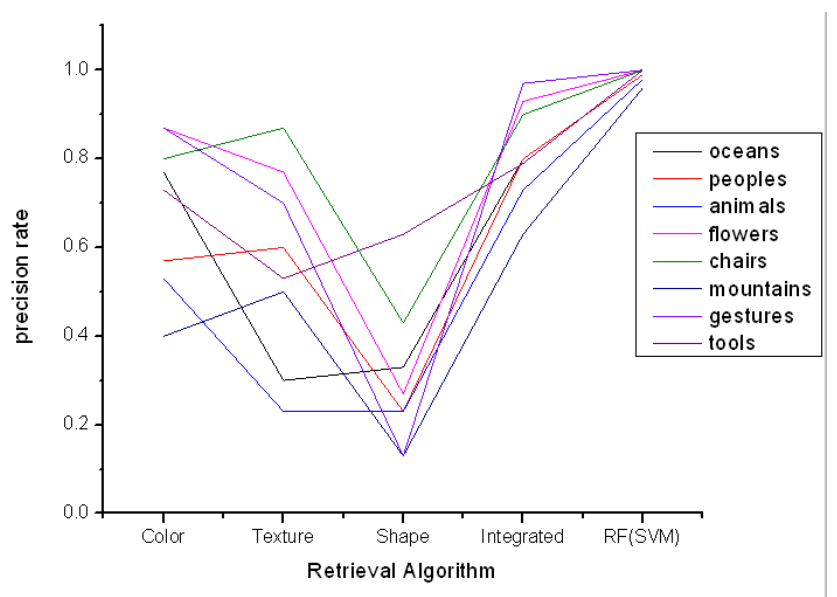

Fig2. Precision rate of different retrieval algorithms

$$
P=p(A \mid B)=\frac{p(A \cap B)}{p(B)}
$$

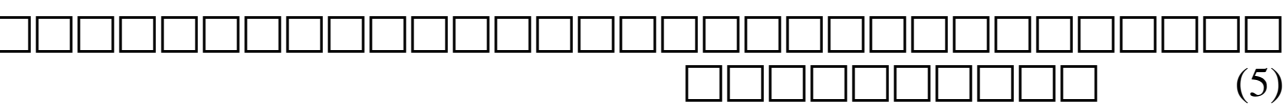

In formula (5), A represents relevant images set, B represents result images set.

Through Analyzing Fig.2, we can conclude:

For every category image, after several (no more than 3 times ) SVM-based reference feedbacks, the precision rate is very satisfied and almost reaches to $100 \%$. So SVM-based reference feedback is a very effective means for user to interact with image retrieval system.

Exp2: In this experiment we use a medical[8] image database. The image database has more than 7000 color gastroscope images. These images include two categories: one category having cancer has 169 images, the other category having no cancer has the rest images.

We choose randomly 10 image from the 169 images having cancer as query images to make the experiment. In every retrieval, according the retrieval results of 20 images, we record the AR of the top 10 result images and the $\mathrm{P}$ rate of the top 15 result images. At the end we get the average AR and $\mathrm{P}$ of all 10 retrievals.

If the top 10 images in result images are relevant, the AR is 5.5 best in theory and the $\mathrm{P}$ is 1 best in theory. After analyzing the result of this experiment, we know the performance of SVM-based relevance feedback is satisfied, the average of AR is 5.6 and the average of $\mathrm{P}$ is 0.92 . while before 2 times of relevance feedback, the AR is 5.77 and the $\mathrm{P}$ is 0.87 .

It seems that SVM-based relevance feedback is very effective to an image retrieval system. Following Figs can explain in more detail. In Fig.3 the $\mathrm{P}$ is 0.6 and AR is 9.5, and 2th,4th,6th,8th,9th,14th,16th,17th are irrelevant images, the rest are relevant images. After 2 times of SVM-based relevance feedback, the result is shown in Fig.4, the P is 1 and AR is 5.5, and only the 17 th is a irrelevant image, all others are relevant images.

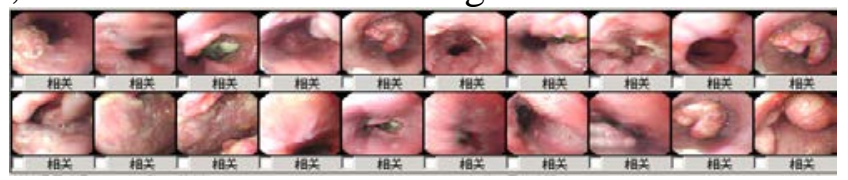

Fig3. Before SVM-based Relevance Feedback

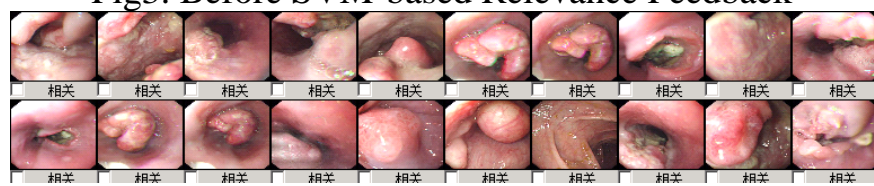

Fig4. After 2 times of SVM-based Relevance Feedback 


\section{Summary}

Through above experiments, we conclude that: relevance feedback is an effective technique in image retrieval, and SVM-based relevance feedback is feasible and effective.

\section{References}

[1] Datta, R., et al., Image retrieval: Ideas, influences, and trends of the new age. ACM COMPUTING SURVEYS, 2008. 40(52).

[2] Deselaers, T., D. Keysers and H. Ney, Features for image retrieval: an experimental comparison. INFORMATION RETRIEVAL, 2008. 11(2): p. 77-107.

[3] hou, X.S. and T.S. Huang, Relevance feedback in image retrieval: A comprehensive review. MULTIMEDIA SYSTEMS, 2003. 8(6): p. 536-544.

[4] Peng, Y., Y. Shang and D. Wang, A novel image retrieval method based on SVM and active learning. Computer Engineering and Science, 2014. 36(7): p. 1371-6.

[5] Ma, C., et al., An improved SVM model for relevance feedback in remote sensing image retrieval. INTERNATIONAL JOURNAL OF DIGITAL EARTH, 2014. 7(9): p. 725-745.

[6] Yildizer, E., et al., Integrating wavelets with clustering and indexing for effective content-based image retrieval. KNOWLEDGE-BASED SYSTEMS, 2012. 31: p. 55-66.

[7] Wang, X. and X. Chen, Efficient Image Retrieval Using Support Vector Machines and Bayesian Relevance Feedback. 2012 5TH INTERNATIONAL CONGRESS ON IMAGE AND SIGNAL PROCESSING (CISP), 2012: p. 786-789.

[8] Hwang, K.H., H. Lee and D. Choi, Medical image retrieval: past and present. Healthcare informatics research, 2012. 18(1): p. 3-9. 